\title{
Minimal surfaces with bounded curvature in Euclidean Space
}

\begin{abstract}
MARC SORET
Let $\mathcal{M}$ be the set of surfaces of bounded curvature that are completely and minimally embedded in Euclidean space. By setting a uniform lower bound for the injectivity radius of the normal bundle of any such surface $M \in \mathcal{M}$, we can show that there is an embedded tubular neighbourhood of constant radius around any $M \in \mathcal{M}$. In particular the area growth of $M$ is not more than cubic, and its spherical area growth, as we will prove, not more than linear. This result can be applied to show that $\mathcal{M}$ is compact with respect to convergence on compact sets of the Euclidean space.
\end{abstract}

\section{Introduction.}

This article is related to the problem of proving the uniqueness of the helicoid and plane among all the simply-connected surfaces that are completely and minimally embedded in the Euclidean space.

As this goal seems distant, we will suppose that the surfaces we study have bounded geometry, that is, that the Gauss curvature is bounded. We will denote the set of these surfaces by $\mathcal{M}$.

There is a double reason for this restriction on the curvature.

The first is that, as was recently proved by L. Rodriguez \& H. Rosenberg in $[R R]$, (generalized recently in $[\mathrm{X}]$ by F. Xavier):

Theorem. If $M \in \mathcal{M}$ and it is transverse to one horizontal plane, and if the intersection with this plane consists of a finite number of curves, then $M$ is either a plane or a helicoid.

Second, it turns out that the normal bundles of these surfaces have a lower uniform bound for their injectivity radius. Indeed we found that:

Theorem 2. For each $M \in \mathcal{M}$, there exists a tube of constant radius that is embedded in Euclidean space. The radius is at least equal to $\rho_{e}:=\frac{\sqrt{3}-1}{\|K\|_{\infty}^{1 / 2}}$, where $K$ is the Gauss curvature function on $M$. 
Applying this theorem leads to the straightforward estimation (also reportedly achieved by W. Meeks) of the area growth and the spherical area growth of the surface (the spherical area being the area given on $M$ by the pulled-back metric of its Gauss map $N$ ).

Theorem 3. Any $M \in \mathcal{M}$ has an area growth that is not more than cubic. More precisely, for any domain $S \subset \mathbf{E}^{3}$,

$$
\text { Area }(M \cap S) \leq c_{1}\|K\|_{\infty}^{1 / 2} \operatorname{Vol}\left(S_{\epsilon}\right)
$$

where $S_{\epsilon}$ is an $\epsilon$-neighbourhood of $S, \epsilon \geq \rho_{e}$ and $c_{1}=\frac{3(2 \sqrt{3}-5 / 2)}{4}$. In particular, the area of $M \cap B(p, r)$ is less than $\pi(2 \sqrt{3}-5 / 2)(r+\epsilon)^{3}\|K\|_{\infty}^{1 / 2}$.

( $S_{\epsilon}$ is the Minkowski sum $S+B_{\epsilon}$ of $S$ and the $\epsilon$-ball around 0 ).

In the case of embedded finite total curvature minimal surfaces with $n$ ends, it is known that $A(M \cap B(p, r)) \leq n \pi r^{2}$. Also note that the cubic area growth is optimal: the helicoid takes a cubic area growth.

Moreover, with the co-area formula as it is applied in [SS], (see also [C]), we establish an estimate of the growth of the total curvature in terms of the growth of the area:

Theorem 4. The Gauss map $N: M \longrightarrow \mathbf{E}^{3}$ has a spherical area growth that is not more than linear: for any $r$-ball around $p \in M$, the spherical area of the connected component of $M$ containing $p$ satisfies $N\left(M_{p} \cap B(p, r)\right) \leq$ $C\|K\|_{\infty} r$, where $C \in \mathbb{R}^{+}$is a constant independent of $M$.

In particular the Gauss map is at most of order one (as defined by Nevanlinna). Notice, for example, that the helicoid is conformally $\mathbb{C}$, and that, when $\mathbb{C}$ is adequately parametrized, its Gauss map, postcomposed with stereographic projection, is the exponential map.

It follows directly from these estimates that $\mathcal{M}$ is compact with respect to the convergence on compact domains of $\mathbf{E}^{3}$.

Finally, before giving the The general outline of the paper, I would like to thank Martin traizet for helpful remarks.

Section 1: We first consider minimal sections that lie in a sufficiently small $\epsilon$-tube of a minimal surface. We prove that for each of these graphs, the domain of definition of these graphs is weakly stable and that their boundary at infinity has zero measure. Here is the definition of weak stability: A surface $M$ is weakly stable if the quadratic form

$$
A(h):=\int_{M}\left(|\nabla h|^{2}+\alpha K h^{2}\right) d A
$$


which is a modified second derivative of the area functional, is positive for any $h \in C_{0}^{2}(M)$, where $\alpha$ is any positive number strictly larger than one.

Proposition 2. Let $D$ be a minimal surface with boundary and let $D^{\prime}$ be a minimal graph above $D$ given by a strictly positive section of the normal bundle to $D$; and suppose $D$ and $D^{\prime}$ have bounded curvature. If $\phi\|K\|_{\infty}^{1 / 2} \leq$ $\beta<\frac{\sqrt{2}}{10}$, then $D$ is weakly stable.

Next, following [CS], we show that $D$ is parabolic, in the sense that its boundary at infinity has zero measure. (This has been proved for the case in which $D$ is complete without boundary in $[\mathrm{H}]$.)

Section 2: We can then prove, in $\S 2.1$, a maximum principle for minimal graphs $\phi$ if $\phi \leq \rho_{e}$. Next we prove in $\S 2.2$, the following maximum principle.

Corollary 3.3. If $M$ and $M^{\prime}$ are two complete embedded minimal surfaces with bounded curvature and $M \cap M^{\prime}=\emptyset$, then $\operatorname{dist}\left(\partial M, M^{\prime}\right)>0$ and $\operatorname{dist}\left(\partial M, M^{\prime}\right)>0$, together, imply that $\operatorname{dist}\left(M, M^{\prime}\right)>0$.

This corollary generalizes a previous result stated in [S]. The key proposition is:

Theorem 1. Let $D$ be a minimal surface with boundary and let $D^{\prime}$ be a minimal graph above $D$ given by a strictly positive section of the normal bundle to $D$; suppose $D$ and $D^{\prime}$ have bounded curvature and that the boundary of $D$ has a geodesic curvature bounded from above, the normal vector to the boundary pointing inwards. If $\phi \leq C$ for some constant given in Proposition 2 then $D$ is parabolic.

Theorem 1 leads directly to the proof that the $\epsilon$-tube of any $M \in \mathcal{M}$ is embedded, as stated in Theorem 2 above. From this we will obtain in $\S 3.2$ the uniform bound for the area as well as a cubic area growth for surfaces in $\mathcal{M}$.

Section 3: In Theorem 3, we use the co-area formula to prove, an a priori uniform bound on the spherical area growth of any $M \in \mathcal{M}$.

Section 4: In this section, we illustrate the usefulness of the uniform area bound. Using a result of H.I. Choi and R. Schoen [CS], (see also B. White $[\mathrm{W}]$ and $\mathrm{A}$. Ros $[\mathrm{R}]$ ), we prove the strong compactness of $\mathcal{M}$. Any two surfaces of $\mathcal{M}$ will be identical if one is carried onto the other by either a rigid motion or a homothety of $\mathbf{E}^{3}$. We can thus normalize any $M \in \mathcal{M}$ so that the curvature verifies $-1 \leq K$. 
Theorem 5. For any sequence of minimal surfaces in $\mathcal{M}$ with at least one accumulation point in $\mathbf{E}^{3}$, a sub-sequence converges in $\mathcal{M}$ on all compacts sets of $\mathbf{E}^{3}$.

\section{Stability of Minimal Graphs.}

Let $\nu(M)$ be the normal bundle of $M \in \mathcal{M}$. With the exponential map, we realize an immersion $\exp : \nu(M) \longrightarrow E^{3}$ in, at least, a neighboorhood of the zero section of $\nu(M)$ (which is diffeomorphic to $M$ and thus identified with $M$ ). The biggest neigborhood $U$ such that exp is an immersion, will be called the normal tube of $M$.

\subsection{Graphs of Minimal Surfaces.}

Let $X: \mathbb{C} \supset U \longrightarrow \mathbf{E}^{3}$ be a minimal immersion of a subset such that the image $X(U)$ is a minimal surface $\Sigma . \quad N: U \longrightarrow \mathbb{S}^{2}$ will denote the Gauss map of $\Sigma$. Let $\bar{X}: U \longrightarrow E^{3}$ be a second immersion whose image, $\bar{X}(U)=\bar{\Sigma}$, is a minimal surface. Furthermore suppose $\bar{\Sigma}$ is a graph above $\Sigma$ that is, a positive section of $\nu(\Sigma)$. A function $\phi$ then exists on $U$ such that

$$
\bar{X}=X+\phi N .
$$

Since the immersions $X$ and $\bar{X}$ are harmonic for each induced metric,

$$
\left\{\begin{array}{l}
\Delta X=0, \\
\bar{\Delta}(X+\phi N)=0 .
\end{array}\right.
$$

In particular, $\phi$ is a minimal graph if

$$
\langle N, \bar{\Delta}(X+\phi N)\rangle_{\mathbf{E}^{3}}=0 .
$$

A direct computation (see $[\mathrm{S}]$ ) gives

Proposition 2.1. Let $\Sigma$ be a minimal surface immersed in a flat 3-manifold of Gauss curvature $K$, and Gauss map $N: \Sigma \longrightarrow \mathbf{S}^{2}$. Let $\nabla$ denote the connection for the metric $g$ of $\Sigma$, and $\bar{\Delta}$, the Laplacian of the pulled-back metric $\bar{g}$ of $\bar{\Sigma}$ induced on $\Sigma$ by $\phi$. Suppose $\phi \in C^{2}(\Sigma, \mathbb{R})$ is a section of $\Sigma$ defining a minimal surface $\bar{\Sigma}$ above $\Sigma$. Then,

$$
\bar{\Delta} \phi+\beta^{2}\left(A(\nabla \phi, \nabla \phi)+K \phi|\nabla \phi|^{2}\right)-2 K \beta^{2}\left(1+K \phi^{2}\right) \phi=0,
$$


where $\beta$ is the ratio of the area elements:

$$
\beta^{-1}=\frac{d A_{\bar{\Sigma}}}{d A_{\Sigma}}=\sqrt{\frac{\operatorname{det} \bar{g}}{\operatorname{det} g}}=\sqrt{\left(1+K \phi^{2}\right)\left(1+K \phi^{2}+|\nabla \phi|^{2}\right)+2 \phi A(\nabla \phi, \nabla \phi)} .
$$

$A$ is the second fundamental form of $\Sigma$ and $\bar{\Delta} \phi=\frac{1}{\sqrt{\bar{g}}}\left(\sqrt{\bar{g}} \cdot \bar{g}^{i j} \phi_{j}\right)_{i}$

Notice that the former equation is uniformly quasilinear elliptic if the minimal graph strictly lies in the normal neighbourhood of $\Sigma$, i.e., if $|\phi(x)| \leq a<\frac{1}{\sqrt{-K(x)}}$. At focal points of the normal bundle, the ellipticity of the minimal surface equation degenerates. Notice too that the linearized equation is the Jacobi equation

$$
\Delta_{\Sigma} \phi-2 K \phi=0
$$

related to the second derivative of the area functionnal (see next definition). subsectionWeak Stability of Minimal Graphs

We recall the definition given in the introduction.

Definition 2.2. A minimal surface $M$ is weakly-stable if the quadratic form

$$
A(h):=\int_{M}\left(|\nabla h|^{2}+\alpha K h^{2}\right) d s
$$

is positive for any $h \in C_{0}^{2}(M)$, where $\alpha$ is some positive number $\alpha>1$.

Next we prove the weak stability of a minimal graph above a domain $D \subset \Sigma$ obtained as follows: take a sufficiently small $\epsilon$-tube of a surface $M \in \mathcal{M}$ that is a tubular neighbourhood of the zero section of the normal bundle; suppose $\Sigma$ intersects this tube away from the zero section. The ensuing estimate defines a minimal section $\phi$ over a domain $D$ of $\Sigma$ that is not necessarily compact and such that $\phi$, at the boundary, is exactly equal to the radius of the tube.

So we will prove

Proposition 2.3. Let $\Sigma$ and $\Sigma^{\prime}$ be two minimal surfaces with boundary and with curvature bounded by a constant $C_{K} \in \mathbb{R}^{+}$. Suppose $\Sigma^{\prime}$ is a graph above $\Sigma$, i.e., $\Sigma^{\prime}$ is a positive section $\phi$ of the normal bundle $\nu(\Sigma)$, such that, $\|\phi\|_{\infty} \leq \frac{\sqrt{2}}{10 C_{K}^{1 / 2}}$, and such that, at boundary points $\left.\phi\right|_{\partial \Sigma} \geq \frac{\sqrt{2}}{10 C_{K}^{1 / 2}}$. Then $\Sigma$ is weakly stable. 
To prove Proposition 2.3 we need two technical lemma; we first prove a stability result for a simplified minimal graph equation, where we essentially substitute in the second term of equation (2.1), the gradient $\bar{\nabla}$ of the graph $\bar{\Sigma}$, by the gradient $\nabla$ taken on $\Sigma$. Next we show in Lemma 2.4 how this substitution affects the stability.

(For notations in the follow-up see Proposition 1 in $\S 1$.)

Lemma 2.4. Let $\phi$ be a positive bounded function solution of the differential inequality $\bar{\Delta} \phi-c|\nabla \phi|^{2}+V \phi \leq 0$ on any Riemannian domain $D$, where $c$ and $V$ are functions that we may take positive w.l.o.g. Then, if $1-c \phi \geq 0$, for any $\alpha>1$, there exists a positive $h$ that satisfies $\bar{\Delta} h+\frac{V}{\alpha} h \leq 0$.

Proof. As $\phi$ is positive, there is $h$ such that $\phi=h^{\alpha}$, where $\alpha \geq 0$. Plug this expression of $\phi$ in the differential inequality, and take $\alpha \neq 1$. This gives

$$
\alpha \cdot h^{\alpha-1} \cdot \Delta h+\left(\alpha \cdot(\alpha-1) h^{\alpha-2}+\left(\alpha . h^{\alpha-1}\right)^{2}\left(1-c h^{\alpha}\right)\right)|\nabla h|^{2}+V h^{\alpha} \leq 0 .
$$

Divide by $\alpha . h^{\alpha-1}$

$$
\Delta h+\left((\alpha-1) h^{-1}+\left(\alpha . h^{\alpha-1}\right)\left(1-c h^{\alpha}\right)\right)|\nabla h|^{2}+\frac{V}{\alpha} h \leq 0 .
$$

The coefficient of $|\nabla h|^{2}$ is non negative:

$$
(\alpha-1) h^{-1}+\left(\alpha . h^{\alpha-1}\right)\left(1-c h^{\alpha}\right) \geq 0 .
$$

That is

$$
(\alpha-1)+(\alpha . \phi)(1-c \phi) \geq 0
$$

which is true if $\alpha \geq 1$. Then from (1):

$$
\Delta h+\frac{V}{\alpha} h \leq 0 .
$$

This completes the proof of Lemma 2.4.

\subsection{Estimates on $\phi$ and weak stability.}

Before proceeding to the next step, which is Lemma 2.5, we need to estimate terms of the minimal equation.

Now consider the minimal equation (2.1) and express it in terms of the metric on $\mathrm{M}$, i.e., substitute $\bar{\Delta}$ by terms involving only derivatives taken 
on $\mathrm{M}$ with its original metric. We will obtain a differential inequality as in Lemma (2.3).

With the same notations as in $\S 2.1$, it is convenient to take on $U$ the isothermic parametrisation given by the curvature lines of $\Sigma$ :

$$
d s^{2}=\rho^{2}|d z|^{2}=\rho^{2}\left(d x_{1}^{2}+d x_{2}^{2}\right)
$$

or

$$
g_{i j}=\rho^{2} \delta_{i j}
$$

$\left(\operatorname{det} g=\rho^{4}\right)$. By a direct computation of the derivatives of $\bar{X}=X+\phi N$, we obtain, the following metric tensor induced by the immersion $\bar{X}$ :

$$
\bar{g}_{i j}=\frac{\beta^{2}}{\rho}\left(a^{i} \delta^{i j}+\frac{\phi_{i} \phi_{j}}{\rho^{2}}\right)
$$

where

$$
a_{i}=1-K \phi^{2}+2 \kappa_{i} \phi, \quad i=1,2
$$

Or,

$$
\left(\bar{g}_{i j}\right)=\rho^{2}\left(\begin{array}{cc}
(1+\kappa \phi)^{2}+\frac{\phi_{1}^{2}}{\rho^{2}} & \frac{\phi_{1}}{\rho} \cdot \frac{\phi_{2}}{\rho} \\
\frac{\phi_{1}}{\rho} \cdot \frac{\phi_{2}}{\rho} & (1-\kappa \phi)^{2}+\frac{\phi_{2}^{2}}{\rho^{2}}
\end{array}\right),
$$

where $\kappa$ is the function $\sqrt{|K|}$.

Note that this is the metric on the the surface obtained by pushing $M$ along the normals at a constant height $\phi(x)$ and then stretched in the direction of the gradient of $\phi$ that takes in account the tilting of $\bar{M}$ at point $\mathrm{x}$.

Suppose the minimal graph lies in the normal tube, i.e., $\inf _{\Sigma}\left(1+K \phi^{2}\right)>$ 0 . Then the minimal graph equation on $M(2.1)$, reads

$$
\bar{\Delta} \phi+\beta^{2}\left(\sum_{i=1}^{2} \kappa_{i}\left(1-\kappa_{i} \phi\right) \frac{\phi_{i}^{2}}{\rho^{2}}\right)-2 K \beta^{2}\left(1+K \phi^{2}\right) \phi=0
$$

where $\kappa_{1}=-\kappa_{2}=\kappa$. In particuliar, we deduce the inequality

$$
\bar{\Delta} \phi-\tilde{c} \beta^{2}|\nabla \phi|_{g}^{2}-2 K \beta^{2}\left(1+K \phi^{2}\right) \phi \leq 0
$$

where $\tilde{c}=\kappa(1-\kappa \phi)$. We won't need to compute explicitely $\bar{\Delta} \phi$ in terms of the laplacian and gradient on $\mathrm{M}$, where

$$
\bar{\Delta} \phi=\frac{1}{\sqrt{\bar{g}}} \partial_{i}\left(\sqrt{\bar{g}} \bar{g}^{i j} \phi_{j}\right)
$$


but only

$$
\langle\bar{\nabla} h, \bar{\nabla} h\rangle_{\bar{g}} .
$$

But, from (2.5) and the above expression of $g_{i j}$ in (2.5)

$$
\left(\bar{g}^{i j}\right)=\frac{\operatorname{det} \bar{g}}{\operatorname{det} \bar{g}} \frac{1}{\rho^{2}}\left(\begin{array}{cc}
(1-\kappa \phi)^{2}+\frac{\phi_{2}^{2}}{\rho^{2}} & -\frac{\phi_{1}}{\rho} \cdot \frac{\phi_{2}}{\rho} \\
-\frac{\phi_{1}}{\rho} \cdot \frac{\phi_{2}}{\rho} & (1+\kappa \phi)^{2}+\frac{\phi_{1}^{2}}{\rho^{2}}
\end{array}\right) .
$$

Or

$$
\bar{g}^{i j}=\frac{\beta^{2}}{\rho}\left(a^{i} \delta^{i j}+\frac{\phi_{i} \phi_{j}}{\rho^{2}}\right) .
$$

Hence

$$
\begin{aligned}
\langle\bar{\nabla} h, \bar{\nabla} h\rangle_{\bar{g}} & =-\bar{g}^{i j} h_{i} h_{j} \\
& =\beta^{2}\left(1+K \phi^{2}\right)\langle\nabla h, \nabla h\rangle-2 \phi A\left(\langle\nabla h, \nabla h\rangle+\langle\nabla h, J \nabla \phi\rangle^{2}\right)
\end{aligned}
$$

Estimates on $\phi, \phi_{i}, \phi_{i j}, A, A_{i}$.

The condition of Lemma 2.4: $1-c \phi>\alpha$ is satisfied if $\tilde{c} \gamma \phi<1-\alpha$, that is if $\kappa \phi<1-\alpha$; geometrically, this is satified if the positive function $\phi$ represents a minimal section that lies inside the half-tube around $\Sigma$ of radius less than $(1-\alpha)$-times the radius of the normal tube.

But we can choose a smaller tube around a domain of $M$.

Consider the $\epsilon$-tube $T(\epsilon, \Sigma)$ with $\epsilon \leq \frac{\nu}{\|K\|_{\infty}^{1 / 2}}$, where $0<\nu<1$. Suppose this tube is not embedded; then pieces of $\Sigma$ will cut the tube away from the zero section. Let $\Sigma^{\prime}$ be one such piece. Note that the boundary of $\Sigma^{\prime}$ consists of points that lie on the boundary of the tube, so that the height at these points equal $\frac{\nu}{\|K\|_{\infty}^{1 / 2}}$.

This piece is a priori not a graph on $\Sigma$ or on a subdomain of $\Sigma$. However we will show this is the case:

Consider a point $p^{\prime} \in \Sigma^{\prime}$ which is above a point $p \in \Sigma$, and at a height less than $\frac{\nu}{\|K\|^{1 / 2}}$ along the normal $p \in \Sigma$. $\Sigma^{\prime}$ is pinched at $p^{\prime}$ between two tangent spheres of radius $\frac{1}{\|K\|^{1 / 2}}$. A straightforward computation shows that these tangent spheres will cut a third sphere with the same radius but tangent to $\Sigma$ at $p$ and below $\Sigma$, if

$$
|\nabla \phi|^{2} \geq\left(\frac{2(1+\nu)}{(1+\nu)^{2}-3}\right)^{2}-1
$$

But then $\Sigma^{\prime}$ would cut $\Sigma$. 
In particular in the $\epsilon$-tube $T(\epsilon, \Sigma)$ with $\epsilon=\frac{\nu}{\|K\|_{\infty} 1 / 2}<\frac{\sqrt{3}-1}{\|K\|_{\infty} 1 / 2}, \Sigma^{\prime} \cap$ $T(\epsilon, \Sigma)$ is a graph above $\Sigma$, and defines a $C^{1}$-bounded minimal graph $\phi$ with

(1) $\|\phi\|<\frac{\sqrt{3}-1}{\|K\|_{\infty}^{1 / 2}}$

(2) $\|\nabla \phi\|^{2} \leq\left(\frac{2(1+\nu)}{(1+\nu)^{2}-3}\right)^{2}-1$.

Hence for a tube sufficiently small we can suppose that

$$
|\phi| \leq \epsilon_{1},|\nabla \phi| \leq \epsilon_{2}
$$

uniformly on the domain of definition of $\phi$ and this estimate is valid up to the boundary of $M$.

(Note that we can obtain estimates for derivatives of $\phi$ of second order : From the bounded geometry hypothesis we assume that the curvature of the surface is uniformly bounded

$$
|A| \leq C_{1}
$$

i.e.,

$$
-K \leq C_{1}^{\prime}
$$

But then, the bound on A implies that locally the surface is given as a graph of a function $\mathrm{u}$ with bounded gradient. Since $|D u|$ is bounded, the minimal surface equation is uniformly elliptic. By standard elliptic theory, we get $C^{\infty}$ estimates for u.Since $|D u|$ is bounded, $\left|D^{3} u\right|$ is proportional to $|D A|$ so we get uniform bounds on $|D A|$. Trivially, $D|A| \leq|D A|$. So

$$
|\nabla A| \leq C_{2}^{\prime}
$$

i.e.,

$$
|\nabla \kappa| \leq C_{2} .
$$

Finally we can likewise deduce a bound on the norm of the second derivatives of $\phi$ from the bounds $|\phi| \leq \epsilon_{1},|\nabla \phi| \leq \epsilon_{2}$, away from the boundary of M.

$$
\left.\left|\phi_{i j}\right| \leq \epsilon_{3}\right)
$$

With the estimates of $\phi_{i}$ we can prove the following result.

Lemma 2.5. If there is a positive function $f$ on $\Sigma$ such that $\bar{\Delta} f+V f \leq 0$. Then there exists a positive function $\psi$ and a constant $\frac{1}{2}<\lambda$ such that $\Delta \psi-2 \lambda K \psi \leq 0$ on $\Sigma$. 
Proof. Suppose there exists a positive $f$ such that

$$
\bar{\Delta} f+V f \leq 0 .
$$

It follows that the first eigenvalue of this operator is positive;

$$
\int_{\Sigma}\left(|\bar{\nabla} h|_{\bar{g}}^{2}-V h^{2}\right) d A_{\bar{g}} \geq 0
$$

for all $h \in C_{0}^{1}(D)$. But it is clear from (2.7) that

$$
|\bar{\nabla} h|_{\bar{g}}^{2} d A_{\bar{g}}=\beta^{2}\left(\left(1-K \phi^{2}\right)|\nabla h|^{2}+\langle\nabla h, \nabla \phi\rangle^{2}+\phi A(\nabla h, \nabla h)\right) \cdot \frac{\operatorname{det} \bar{g}}{\operatorname{det} g} \cdot d A_{g} .
$$

But $\operatorname{det} \bar{g}=\frac{\operatorname{det} g}{\beta^{2}}\left(\beta\right.$ is given explicitely in Proposition 1.1), and $-K \phi^{2}=$ $\kappa^{2} \phi^{2}$; hence

$$
|\bar{\nabla} h|_{\bar{g}}^{2} \leq\left(\left(1+\kappa \phi+\kappa^{2} \phi^{2}+|\nabla \phi|^{2}\right)|\nabla h|^{2} .\right.
$$

It is immediate that

$$
\left\{\begin{array}{l}
\int_{\Sigma}|\bar{\nabla} h|^{2} d A_{\bar{g}} \leq \delta \cdot \int_{\Sigma}|\nabla h|^{2} d A_{g} \\
\delta=\left\|1+\kappa \phi+\kappa^{2} \phi^{2}+|\nabla \phi|^{2}\right\|_{\infty}
\end{array}\right.
$$

Thus, from (2.10)

$$
\delta \int_{\Sigma}|\nabla h|^{2} d A_{g}-\int_{\Sigma} \frac{V}{\beta^{2}} h^{2} d A_{g} \geq 0
$$

This simplifies to

$$
\int_{\Sigma}|\nabla h|^{2} d A_{g}+\frac{2}{\delta} \int K\left(1+K \phi^{2}\right) h^{2} d A_{g} \geq 0
$$

if we use the expression of $V$ given in (2.6). But as we chose the tube small such that $\kappa \phi \leq 1-\alpha$ so that

$$
\left\{\begin{array}{l}
1+K \phi^{2}>\alpha(2-\alpha) \\
\delta \leq 3-3 \alpha+\alpha^{2}+\|\nabla \phi\|_{\infty}^{2} .
\end{array}\right.
$$

Consequently, $\Sigma$ is weakly stable if ( $K$ has a negative sign)

$$
\frac{2 \alpha(2-\alpha)}{\delta} \geq 1
$$

that is if

$$
\|\nabla \phi\|_{\infty}^{2} \leq \frac{\alpha}{3}-(\alpha-1)^{2} .
$$

A direct computation shows that (2.9) together with (2.10) are satisfied if $\alpha>1-\sqrt{2} / 10$, i.e. if $\nu<\sqrt{2} / 10$; this is a rough estimate but sufficient for our purpose. 


\subsection{Parabolic minimal domains.}

We now prove the parabolic nature of $\mathrm{M}$ (or $\bar{M}$ ) as described as above, ie, a piece of minimal surface which is the support of the function $\phi$ that defines a graphical surface $\bar{M}$. Moreover, proposition 2.3 shows that $M$ (and $\bar{M}$ ) are weakly stable. Hence $M$ is clearly parabolic when $M$ has no boundary (cf. [FC]). More generally we prove that, when $\partial M$ is not empty, $M$ is still parabolic,that is: if $u$ is a bounded continuous function which satisfies $u \leq c$ on $\partial M$ and such that $\Delta u \geq 0$ on $M$, then $u \leq c$ on $M$. (in other words the maximum principle applies to continuous bounded subharmonic function) The proof is in two parts; in the second part (proposition 2.11) we show the parabolic nature of a domain $N$ whose boundary has geodesic curvature bounded from above, such that there is positive $u$ that satisfies $\Delta u-2 \alpha K u \leq 0$, and that is bounded from below on the neighborhood of $\partial N$ ( for example $u \geq 1$ if $\operatorname{dist}(x, \partial N) \leq \epsilon$ ). In the first part we show that such a positive $u$ exists. Notice that, from Lemma 2.4, we already know that there exists a positive function $u_{0}$ that is, by construction, uniformly bounded from below on $\partial M$; however $u_{0}$ satisfies an equality of the ' mixed type" $\bar{\Delta} u_{0}+\alpha K u_{0} \leq 0$, with the metric of $\bar{M}$ and the curvature of $M$ ( or resp. $\Delta v_{0}+\alpha \bar{K} v_{0} \leq 0$ for a positive $v_{O}$ with uniform bound on the boundary)

We will, in the first part, estimate $|K-\bar{K}|$ and then show that the existence of $u_{0}$ ( or $v_{0}$ ) implies the existence of a positive $u$ with a uniform bound from below at the boundary and solution of

$$
\bar{\Delta} u+\alpha \bar{K} u \leq 0
$$

We now come to the heart of the matter:

Claim. There exists a positive function $u$ on $M$, bounded from below on a tubular neighborhood in $M$ of $\partial M$, such that

$$
\bar{\Delta} u+\beta \bar{K} u \leq 0, \quad \beta<-1 .
$$

Let us first estimate $|K-\bar{K}|$.

Proposition 2.6. Let $M$ and $\bar{M}$ be two weakly stable pieces of minimal surfaces of bounded curvature and let $\bar{M}$ a minimal surface over $M$ whose distance to each point of $M, \phi$, is uniformly bounded and whose first derivative is uniformly bounded by $\epsilon$. Let $K_{M}$ and $K_{\bar{M}}$ be respectively the Gaussian 
curvature function on $M$, respectively on $\bar{M} . K_{\bar{M}}$ defines a function on $M$ : $\bar{K}(q)$, the Gaussian curvature of the point of $\bar{M}$ above $q)$. There exists $0<\alpha<1$ such that for any point $p \in M, M$ and $\bar{M}$ are graphical over a flat tangent disk $\tilde{D}_{\alpha r / 2}(p)$ around $p$ of radius $\frac{\alpha r}{2}$ where $r:=\operatorname{dist}_{M}(p, \partial M)$. Moreover there exist constant $C_{4}$ and $C_{4}^{\prime}$, independant of $\phi$, (but dependent on $\epsilon$ ), such that

$$
\begin{aligned}
\sup _{q \in \tilde{D}}\left(\frac{|\nabla \phi(q)|}{\phi(q)}\right) & \leq \frac{C_{4}}{r} \\
\sup _{q \in \tilde{D}}\left|K_{M}(q)-K_{\bar{M}}(q)\right| & \leq \frac{C_{4}^{\prime} \sup _{\tilde{D}} \phi}{r^{3}} .
\end{aligned}
$$

Proof. Consider a geodesic disk of radius $r$ around $p, D_{r}(p)$ in $M$. From [SS] we obtain the curvature estimate $K(q) \leq \frac{C}{r^{2}}$ for any $q \in D_{r / 2}(p)$,. Consequently on the subdisk $D_{\alpha r / 2}(p)$, where $0<\alpha \leq 1$ independently of $p$, the variation of the Gauss map is bounded:

$$
|\nabla N(q)| \leq \frac{C^{\prime}}{r} \text { hence }|N(q)-N(p)| \leq 2 C^{\prime} \alpha, \quad \forall q \in D_{\alpha r / 2}(p) .
$$

Thus, for $\alpha<1$ sufficiently small, a piece of $M$ is graphical over the flat disk $\tilde{D}_{\alpha^{\prime} r / 2}(p)$.

Furthermore, the hypothesis on $\phi$ and its derivatives show that $\bar{M}$ is also graphical over $\bar{D}_{\alpha r / 2}(p)$.

We then translate this piece of $M$ and $\bar{M}$ that are graphical over $\bar{D}_{\alpha r / 2}(p)$ from $p$ to a fixed origin and then rescale by dilating $\mathbb{R}^{3}$ by a ratio $\frac{2}{\alpha r}$; we thereby obtain two pieces of graphical surfaces over a disk of radius 1 with uniformly bounded gradients and curvature.

Lemma 2.7. Let $f$ and $g$ two functions of minimal graphs over a flat disk of radius 1 whose $C^{2}$-norm of these graphical minimal surfaces are bounded. Then there exist constants $C_{2}$ and $C_{2}^{\prime}$ independent of $f$ and $g$ such that on $D_{1 / 2}$,

$$
\begin{aligned}
\left\|f_{i j}-g_{i j}\right\|_{0} & \leq C_{2}\|f-g\|_{0} \\
\left\|\frac{\nabla(f-g)}{(f-g)}\right\|_{0} & \leq C_{2}^{\prime} .
\end{aligned}
$$


Let $L$ be the minimal operator defined by

$$
L(f)=\left(1+f_{x}^{2}\right) f_{y y}-2 f_{x} f_{y} f_{x y}+\left(1+f_{y}^{2}\right) f_{x x} .
$$

Let $t$ be a function of $x$ and consider:

$$
L(f+t(g-f)) .
$$

This function is smooth with respect to $t$. Because

$$
L(f)=L(g)=0,
$$

there exists a smooth function $t_{0}(x)$ such that

$$
L^{\prime}\left(f+t_{o}(g-f)\right) \cdot(g-f)=0 .
$$

Moreover $M=L^{\prime}\left(f+t_{o}(g-f)\right)$ is an order 2 uniformly elliptic linear operator of the type

$$
M u=c_{i j} u_{i j}+c_{i} u_{i},
$$

where $u:=g-f$, and the coefficients $c_{i j}$ are uniformly bounded, that is $c_{i j}$ and $c_{i}$ are functions of first derivatives of $f$ and $g$ that are uniformly bounded because the curvatures of the surfaces are bounded.

We apply Schauder estimates to this equation (cf. [GT]) and deduce the existence of a constant $C_{2}$ independant of $f$ and $g$ such that

$$
\left\|u_{i j}\right\| \leq C_{2}\|u\| .
$$

This proves in particular the first inequality of lemma 2.7 .

To obtain the second inequality, we apply the gradient interior estimate of [CY] for the function $\log u$.

We have so far obtained an estimate of the difference in height between the rescaled pieces and the flat disk, $u=f-g$. The distance $\phi_{-}$from the piece of $M_{f}$ to the piece of $M_{g}$ can be expressed in terms of $u$ : take coordinates $(x, y, z) \in \mathbb{R}^{3}$ such that the flat disk is parametrized by $(x, y):=$ $(x, y, 0)$. From the mean value theorem, we see that

$$
u(x, y):=\phi_{-}(x, y) \zeta(x, y)
$$

where

$$
\zeta=\left(N_{3}(x, y)-N_{1}(x, y) g_{x}^{\prime}(\xi, \mu)-N_{2}(x, y) g_{y}^{\prime}(\xi, \mu)\right), \quad(x, y),(\xi, \mu) \in D_{1 / 2} .
$$


where $N$ is the normal to $M_{f}$ at point $(x, y, f(x, y)$. $\zeta$ is bounded in the $C^{2}$-norm and if the bound on the first derivatives, $\epsilon$ is small enough, then $\zeta$ is bounded from below by a positive constant. Thus, from (2.12) we see that in particular that

$$
\left\|K_{f}-K_{g}\right\|_{0} \leq C_{3}\left\|\phi_{-}\right\|_{0} \text { and }\left\|\frac{\nabla \phi_{-}}{\phi_{-}}\right\|_{0} \leq C_{2}^{\prime \prime} .
$$

We finally replace the expression of the curvature and gradient of the retracted graphs in terms of the original surfaces before retraction. This gives Proposition 2.6.

Proposition 2.8 (solution to the perturbed inequality). Suppose there exists on $M$ a function $u_{0}>0$ such that

$$
\Delta u_{0}+V u_{0} \leq 0
$$

where $V>0$ is a potential which decays quadratically; let $\lambda$ be a positive function on $M$ such that

$$
\lambda(p) \leq \frac{C_{3}}{r^{3}} \quad r:=\operatorname{dist}(p, \partial M) .
$$

Accordingly there exists a positive number $r_{0}$, as well as a positive function $u$, such that,

$$
\Delta u+(V+\lambda) u \leq 0
$$

on the subdomain $M_{r_{0}}$ of points whose distance to the boundary of $M$ is at least $r_{0}$.

Proof. Let us begin by reviewing three properties of $u_{0}$ : first, from Lemma $2.4, u_{o}=\phi^{\alpha}$; because $\phi$ is positive and equal to one on the boundary, so is $u_{0}$.

Second, the derivatives of $\phi$ are uniformly bounded; in particular $u_{0}$ is bounded below by a positive constant on the tubular neighborhood of $\partial M_{1}$.

Third, from proposition (2.6), there is a constant $C_{4}$ such that for $p \in D$,

$$
\left\|\frac{\nabla u_{0}}{u_{0}}\right\|_{0, D} \leq \frac{C_{4}}{r}
$$

where $D$ is the geodesic disk of radius $\alpha r$ around $p$ for a positive $\alpha<1$. 
We can now solve (2.13).

Define

$$
w=: \frac{u}{u_{0}}
$$

Then

$\frac{1}{u_{0}}(\Delta u+V u)=\frac{1}{u_{0}}\left(\Delta\left(w u_{0}\right)+V w u_{0}\right)=\Delta w+2\left\langle\nabla w, \frac{\nabla u_{0}}{u_{0}}\right\rangle+\frac{w}{u_{0}}\left(\Delta u_{0}+V u_{0}\right)$.

Hence, if

$$
L_{0} w:=\Delta w+2\left\langle\nabla w, V_{0}\right\rangle \leq-\lambda w
$$

where $V_{0}:=\frac{\nabla u_{0}}{u_{0}}$, then $(2.13)$ is satisfied.

The proof that a positive solution to (2.13) exists, has been reduced to the proof of the following lemma.

Lemma 2.9. Suppose $\partial M$ has bounded geodesic curvature from above. There exist positive solutions $w$ of

$$
L_{1} w=L_{0} w+\lambda w \leq 0
$$

with

$$
L_{0}=\Delta+\nabla_{V_{0}}, \quad\left|V_{0}\right| \leq \frac{C_{4}}{r} .
$$

Furthermore these solutions are bounded from below on a tubular neighborhood of $\partial M_{r_{0}}$.

We use comparative functions to prove Lemma 2.9.

Proof. Let $p$ be any point in $M$ and consider a minimizing geodesic that joins $p$ to the boundary $\partial M$. In a neighborhood of this geodesic, the metric of $M$ can be displayed in terms of the distance $r$ to the boundary $\partial M$ and of $t$, the normal distance to the geodesic:

$$
d s^{2}=d r^{2}+G(r, t)^{2} d t^{2} .
$$

This parametrization of $M$ is smooth up to $p$. For technical reasons, we will take $r \geq 1$, and consider the subdomain $M_{1}$ of $M$ whose points lie at a distance at least 1 from the boundary of $M$. On this subdomain we consider functions $u$ that depend only on $r$; for such functions,

$$
\Delta u=u^{\prime \prime}+u^{\prime}\left(\frac{1}{r}+(\log G)^{\prime}\right)
$$


Notice that $(\log G)^{\prime}=\kappa_{g}(r, t)$ i.e. the geodesic curvature of a parallel curve at distance $r$ from $\partial M$. This means that the geodesic curvature corresponds to the variation of length of the parallel curve to the boundary in the positive $r$ direction, i.e., moving away from the boundary along a geodesic normal to the boundary of $M$.

As the Gaussian curvature $K$ decays quadratically in $r$, we can estimate $\kappa_{g}(r, t)$ in terms of $\kappa_{g}(1, t)$ via the following inequalities

$$
-\kappa_{g}^{2} \leq \frac{d \kappa_{g}(t, r)}{d r}=-K-\kappa_{g}^{2} \leq \frac{C}{r^{2}}-\kappa_{g}^{2} .
$$

We now bound $\kappa_{g}$ from above, (resp. from below), with test-functions $F_{0}$ (resp. $f_{0}$ ) that satisfy

$$
\left\{\begin{array}{l}
F_{0}^{\prime}(r) \geq \frac{C}{r^{2}}-F_{0}^{2} \\
F_{0}(t, 1) \geq \kappa_{g}(t, 1)
\end{array}\right.
$$

and $\kappa_{g}(t, r) \leq F_{0}(t, r)$

(resp.

$$
\left\{\begin{array}{l}
f_{0}^{\prime}(r) \leq-f_{0}^{2} \\
f_{0}(t, 1) \leq \kappa_{g}(t, 1)
\end{array}\right.
$$

and $\left.\kappa_{g}(t, r) \geq f_{0}(t, r)\right)$.

Suppose that boundary has bounded geodesic curvature from above:

$$
\kappa_{g}(t, 1) \leq b_{0} .
$$

Then we check that the function

$$
F_{0}(r)=\frac{b}{r}
$$

with conditions

$$
\left\{\begin{array}{l}
b(b-1)>C \\
b>b_{0}
\end{array}\right.
$$

satisfies inequalities (2.15).

Similarly the function

$$
f_{0}(r)=\frac{1}{\frac{1}{\kappa_{g}(t, 1)}+r-1}
$$

satisfies (2.16). 
Let us consider separately the two following cases:

In the first case, when $\kappa_{g}(t, 1) \geq 0$, notice that, as a result of $(2.15)$ and (2.16), we obtain:

$$
\frac{1}{\frac{1}{\kappa_{g}(t, 1)}+r-1} \leq \kappa_{g}(r, t) \leq \frac{b}{r} \quad \forall r>1 .
$$

In particular

$$
\left|\kappa_{g}(r, t)\right| \leq \frac{b}{r}
$$

In the second case, when $\kappa_{g}(t, 1)<0$, notice that $(2.16)$ holds for $r \leq$ $r_{c}(t)=1-\frac{1}{\kappa_{g}(t, 1)}$, until the geodesic reaches the cut-locus where $\kappa_{g}=-\infty$. However, since the geodesic that joins $p$, which is not on the cut-locus, to the boundary is minimizing, $p$ must lie before the cut-locus, hence $\kappa_{g}(t, r)$ is defined at least up to point $p$ but $r$ extends continuously up to the cut-locus.

In both cases we can always conclude that

$$
\kappa_{g}(r, t) \leq \frac{b}{r}
$$

Now we go back to the operator $L_{1}$, defined in lemma 2.9 and study its operation on a function $u$, smooth on $M \backslash$ (cut locus), and that depends only on the distance to the boundary. As $\kappa_{g}$ and $V_{0}$ are bounded, the inequality above shows that

$$
L_{1} u=\Delta u+\nabla_{V_{0}} u=u^{\prime \prime}+\psi \frac{u^{\prime}}{r}
$$

where $\psi$ is a function defined on $M_{1}$ that is uniformly bounded from above, i.e.

$$
\psi \leq b, \quad b \in \mathbb{R}^{+} .
$$

Lemma 2.10. Let $\psi$ be any function of $r, \psi \leq b, b \in \mathbb{R}$. Then there exist $r_{0}$, and positive smooth functions $u$ of the variable $r$ (distance to the boundary) that satisfy

$$
u^{\prime \prime}+\frac{\psi}{r} u^{\prime}+\frac{C}{r^{3}} u \leq 0 \quad C \in \mathbb{R} \quad r>r_{0}
$$

Proof. The proof is in 3 steps. 
We first solve an ODE ; for $b>1, b \in \mathbb{N}, c_{1} \in \mathbb{R}$.

$$
u^{\prime \prime}+\frac{b}{r} u^{\prime}+\frac{c_{1}}{r^{3}} u=0 .
$$

Solutions are best written in terms of the Bessel functions of the first and second type $J$ and $Y$ (see [WA]):

$$
v=A\left(\frac{1}{\sqrt{x}}\right)^{b-1} J_{b-1}\left(\frac{2 \sqrt{c_{1}}}{\sqrt{x}}\right)+B\left(\frac{1}{\sqrt{x}}\right)^{b-1} Y_{b-1}\left(\frac{2 \sqrt{c_{1}}}{\sqrt{x}}\right) .
$$

Let $A=0, B<0$. Then function $u$ is asymptotically equal to

$$
v \sim v_{\infty}=-B \frac{(b-2) !}{\pi} c_{1}^{\frac{1-b}{2}}>0 .
$$

And $u^{\prime}$ is asymptotically equal to

$$
v^{\prime} \sim \frac{v_{\infty}^{\prime}}{r^{2}}=B \frac{(b-3) !}{\pi r^{2}} c_{1}^{\frac{1-b}{2}} .
$$

Second, for $r$ large, there exist solutions of

$$
u^{\prime \prime}+\frac{b}{r} u^{\prime}+\frac{c_{2}}{r^{3}} u \leq 0 \quad \forall c_{2} \in \mathbb{R}^{+}
$$

with $u>0$ and $u^{\prime}>0$.

Specifically, let $u:=v-\frac{\epsilon}{r}, \quad 0<\epsilon$ Then, for $\mathrm{r}$ large enough, $u>0$ and as $u^{\prime}=v^{\prime}+\frac{\epsilon}{r^{2}}, u^{\prime}>0$ if $\epsilon>v_{\infty}^{\prime}$.

Moreover

$$
u^{\prime \prime}+\frac{b}{r} u^{\prime}+\frac{c_{2}}{r^{3}} u=\frac{\epsilon}{r^{3}}\left(-2+b-\frac{c_{2}}{r}\right)+\frac{c_{2}-c_{1}}{r^{3}} v \leq 0
$$

for $r$ sufficiently large if $c_{1}$ is large i.e., if $c_{1}>c_{2}+\frac{(b-2) \epsilon}{v_{\infty}}$.

Finally if $c_{2}>C$ then

$$
u^{\prime \prime}+\frac{\psi}{r} u^{\prime}+\frac{C}{r^{3}} u \leq 0 .
$$

That is,

$$
u^{\prime \prime}+\frac{\psi}{r} u^{\prime}+\frac{C}{r^{3}} u \leq \frac{(\psi-b)}{r} u^{\prime}+\frac{\left(C-c_{2}\right)}{r^{3}} u .
$$

But, if $\psi-b \leq 0$ and $\left(C-c_{2}\right) \leq 0$,

$$
\frac{(\psi-b)}{r} u^{\prime}+\frac{\left(C-c_{2}\right)}{r^{3}} u \leq 0 .
$$


End of proof of Lemma 2.9 .

Now that we have proved lemma 2.10 we can go back to finish proving lemma 2.9 .

We knew from Lemma 2.10 that the function $\tilde{u}:=u \circ r$ solution of inequality (2.17) is positive, has a positive asymptotic value, and from the expression of the Laplacian and remark preceeding Lemma 2.10, that it satisfies on $M_{r_{0}} \backslash\{$ cut locus $\}$

$$
\Delta \tilde{u}+\nabla_{V_{0}} \tilde{u}+\lambda \tilde{u} \leq 0 .
$$

Notice that the inequality extends to the cut-locus, since $\Delta u \mapsto-\infty$ (see for example $[\mathrm{K}]$ p. 185- ) ; hence, in the sense of distribution

$$
\Delta \tilde{u}+\nabla_{V_{0}} \tilde{u}+\lambda \tilde{u} \leq 0, \quad \text { on } M_{r_{0}}
$$

End of proof of Proposition 2.8 .

We can now finish proving Proposition 2.8.

Let us define $u:=\tilde{u} . u_{0}$; then $u$ is a positive solution of (2.13).

Let us now consider the behavior of $u$ on the boundary of $M_{r_{0}}$. Recall that $u_{0}$ is uniformly bounded from below by a positive constant $c$ in a tubular neighbourhood in $M$ of $\partial M$ of small radius $\epsilon$ (see the beginning of $2.4: u_{0}$ is equal to $c$ on $\partial M$ ant its first derivatives are uniformly bounded). We claim that $u_{0}$ is bounded from below on $\partial M_{r_{0}}$; apply the Harnack inequality on $u_{0}$ on open disks $D_{p}$ around points $p \in \partial M_{r_{0}}$ of radius $r_{0}$. On the concentric subdisk of radius $\frac{r_{0}-\epsilon}{2}, \sup u_{0} \leq K_{D_{p}} \inf u_{0}$. As $M$ has bounded geometry, we may bound uniformly $K_{D_{p}}$ by some constant $C: \sup u_{0} \leq C \inf u_{0}$. In particular, in a tubular neighbourhood of $\partial M_{r_{0}}, u_{0}$ is bounded below by $\frac{c}{C}$.

Finally, as $\tilde{u}$ is a function of $r$ only, $u:=\tilde{u} . u_{0}$, is bounded below in a tubular neighborhood of $\partial M_{r_{0}}$.

Theorem 1. $M$ is par olic.

To prove this theorem, it remains to prove

Proposition 2.11. Let $M$ be a minimal piece whose boundary's geodesic curvature is uniformly bounded from above. Suppose there exists a positive 
function $\phi$ that satisfies $\Delta \phi+\alpha|A|^{2} \phi \leq 0, \quad \alpha>1$ and suppose that $\phi$ is, on a tubular neighborhood of $\partial M$, uniformly bounded from below by a positive constant ; then $M$ is parabolic.

Proof. (We will chose the same convention for the sign of the geodesic curvature as in the proof of Lemma 2.9) Let $\phi \geq 0$ such that $\Delta \phi-\alpha K \phi \leq 0$, with $\alpha>1$, and such that $\phi$ is bounded below in a neighborhood of $\partial M_{r_{0}}$ (Proposition 2.8).

Change the original metric $d s$ on M conformally by the factor $\phi$ that introduces a new metric : $d \tilde{s}=\phi d s$. By proving the parabolicity of $\mathrm{M}$ with this new metric we will prove that $(M, d s)$ is parabolic. We will prove this in 4 steps.

1. $(M, d \bar{s})$ has non negative scalar curvature

We choose local isothermic coordinates on $\mathrm{M}$; then $d s=\lambda|d z|, d \tilde{s}=$ $\lambda \phi|d z|$. Let $\Delta_{0}$ be the flat laplacian.

$$
\tilde{K}=-\frac{\Delta_{0} \log (\phi \lambda)}{\phi^{2} \lambda^{2}}=-\frac{\Delta \log (\phi)}{\phi^{2}}-\frac{\Delta \log (\lambda)}{\phi^{2}} .
$$

But $\Delta \phi-a K \phi \leq 0$, hence

$$
\tilde{K}=-\frac{1}{\phi^{2}}\left(\frac{\Delta \phi}{\phi}-\frac{|\nabla \phi|^{2}}{\phi^{2}}\right)+\frac{K}{\phi^{2}} \geq-\frac{1}{\phi^{2}}\left(a K-\frac{|\nabla \phi|^{2}}{\phi^{2}}\right)+\frac{K}{\phi^{2}} .
$$

But the Gaussian curvature of a minimal surface $K$ is not positive ; thus if $a \geq 1$, then

$$
\tilde{K} \geq 0 \text {. }
$$

2. $\left(M_{r_{0}}, d \tilde{s}\right)$ is complete.

The proof is the same word for word as that given in $[\mathrm{FC}]$. We note that a diverging geodesic starting from point $\mathrm{p}$ that touches the boundary infinitely often must have infinite length, since $\phi$ is bounded from above in a neighborhood of the boundary. Hence we need only consider diverging geodesics that touch the boundary a finite number of times. This boils down to study the case of a diverging geodesic in a complete surface without boundary.

(We may go directly to step 4. and apply it to prove next Proposition 3.2 directly to the subdomain $M_{r_{0}}$ ).

3. If $M_{r_{0}}$ is parabolic then $M$ is parabolic. 
Consider the set of paths in $M$ starting at a point $p \in M_{r_{0}}$. We will show that any such path $\gamma$ will hit $\partial M$ with probability one.

On the first hand, let us consider paths $\gamma$ such that, for some $t_{0}, \gamma(t) \in$ $M_{r_{0}}, \forall t>t_{0}$. As $M_{r_{0}}$ is parabolic, the Wiener measure of the set of such $\gamma$ is zero.

On the other hand, consider paths $\gamma$ that hit $M \backslash M_{r_{0}}$ infinitely often at points $p_{n}, n \in \mathbb{N}$. Let us prove that $\gamma$ will hit $\partial M$ with probability one.

Let $\tau_{0}$ the geodesic of length $d$ joining $\partial M$ to $p_{n}$ and $A B$ a piece of $\partial M$ such that $p_{n}$ is the mid-point. Let $A C$ and $B D$ be 2 geodesics from $\partial M$ of length $2 d$. From Kakutani's result, the Wiener measure of the set of paths that start at $p_{n}$ and hit $A B$ for the first time before hitting $\sigma:=A C D B$ is given by the value of the harmonic function $\omega\left(p_{n}\right)$ where $\omega$ is defined on the domain $R$ bounded by $A B C D$, and equals 0 on $\sigma$ and 1 on the piece of boundary $A B$. Let us find a lower bound of $\omega\left(p_{n}\right)$. This is equivalent to finding an upper bound at $\mathrm{p}$ of the harmonic function $\eta:=1-\omega$, that is, the harmonic function that is 1 on $\sigma$ and 0 elsewhere on the boundary.

One way to estimate the value of the harmonic measure $\eta$ at $\mathrm{p}$ is the notion of extremal length (cf. [AB]) There exists an upper bound on $\eta$ in terms of the extremal length:

$$
\eta\left(p_{n}\right) \leq C e^{-\pi \lambda\left(p_{n}\right)}
$$

which gives a lower bound for

$$
\omega=1-\eta \geq 1-C e^{-\pi \lambda\left(p_{n}\right)}
$$

where

$$
\lambda(p)=\sup _{\tau} \lambda(p, \tau, \sigma, R)
$$

The supremum is taken for all cuts $\tau$ starting from $p_{n}$ and joining $\sigma^{c}=A B$ in $\partial R$ such that $\tau^{c}$ is simply-connected, where $\lambda(p, \tau, \sigma, R)$ is the extremal length in $R$ between $\sigma$ and $\tau$ for the set of all paths joining $\sigma$ to $\tau$. Recall that

$$
\lambda(p, \tau, \sigma, R)=\sup _{\rho} \inf _{\mu} \frac{l_{\rho}^{2}(\mu)}{A_{\rho}(R)} .
$$

The infimum is taken on all paths $\mu$ joining $\tau$ to $\sigma$ in $R$, and the supremum over all Borel functions $\rho$, the area and distance being computed in the metric $\rho . d s$.

Let us choose $\tau=\tau_{0}$, which gives a value $\lambda_{o} \leq \lambda$. Furthermore let $\rho$ be the natural metric on $M$; this gives a lower bound $\lambda_{1}$ for $\lambda_{0}$. Therefore

$$
e^{-\pi \lambda} \leq e^{-\pi \lambda_{0}} \leq e^{-\pi \lambda_{1}}
$$


That is

$$
\omega \geq 1-C e^{-\pi \lambda} \geq 1-C e^{-\pi \lambda_{0}} \geq 1-C e^{-\pi \lambda_{1}} .
$$

We then estimate the area and length on $M$ with comparison theory as in Lemma 2.9. As the curvature of $M$ is bounded, the area $A(R)$ is bounded by a constant $A_{0}$ that depends only on $d$ and $A B$. (From Lemma 2.9 the area is polynomial in $d$, thus $A_{0} \leq C^{\prime} A B d^{n}$.)

Hence

$$
\lambda_{1} \geq \frac{\left(\frac{A B}{2}\right)^{2}}{A_{0}} \geq \frac{C^{\prime \prime} A B}{d^{n}}=C_{5} .
$$

Now let $A B$ be sufficiently big that $C e^{-\pi C_{5}}<1$. We finally obtain a bound from below for $\omega$ independent of $n$.

Consequently $\gamma$ has probability one to hit the boundary since it hits a tubular neighborhood of $\partial M$ infinitely often, and since, at each point $p_{n}$, the probability to hit $\partial M$ is bounded below by a constant independant of $p_{n}$. (In particular $M \backslash M_{r_{0}}$ is parabolic too)

\section{4. $\mathrm{M}$ is parabolic}

The function $\log (\tilde{r}+1)$ is superharmonic on $M$ where $\tilde{r}$ is the distance to the boundary with respect to the new metric. We now prove that the maximum principle for bounded subharmonic function is true. In other words, when $u$ is a bounded subharmonic function such that $\left.u\right|_{\partial M} \leq c$ then we prove that $u \leq c$ on $M$.

Consider $v:=u-\epsilon \log (\tilde{r}+1)$, for some positive $\epsilon$. We can easily verifiy that $v$ is subharmonic and that, for a big $r_{0}, v \leq c$ on $M_{r_{0}}$. But from the proof of step 3, $M \backslash M_{r_{0}}$ is parabolic; hence $v \leq c$ on $\partial\left(M \backslash M_{r_{0}}\right)$ implies $v \leq c$ on $M \backslash M_{r_{0}}$. Thus $v \leq c$ on $M$. That is $u \leq c+\epsilon \log (\tilde{r}+1)$. As this is true for any $\epsilon$, we deduce that $u \leq c$ on $M$.

\subsection{Maximum Principle.}

In $[\mathrm{S}]$, we proved:

Proposition 3.1. Let $\Sigma$ be a complete parab $a$ minimal surface in a complete flat 3-space, and let $\phi$ be a minimal graph bounded in the $C^{1}$-norm, $\phi \geq 0$ on $\Sigma$. If $\phi \geq \delta>0$ on $\partial \Sigma$, then $\phi(x) \geq \min _{\Sigma}\left(c, \frac{1}{\sqrt{|K(x)|}}\right)$ on $\Sigma$.

(Idea of the proof: a positive minimal graph on a surface is subharmonic for a semilinear elliptic equation; and the maximum principle is satisfied for 
subharmonic functions of this semilinear elliptic equation if the domain is parabolic and has bounded curvature).

Notice that it is possible to construct a bounded solution of the minimal equation on a hyperbolic domain that doesn't satisfy the maximum principle. But with the help of Proposition 2.6, and the latter proposition, we will get rid of the parabolicity hypothesis and prove:

Proposition 3.2. Let $\Sigma$ be a complete minimal surface in a complete flat 3 -space, and let $\phi$ be a minimal graph $\phi \geq 0$ on $\Sigma$. If $\phi \geq \delta>0$ on $\partial \Sigma$, then $\phi(x) \geq \min \left(\delta, \rho_{e}\right)$ on $\Sigma$ with $\rho_{e}=\frac{\sqrt{3}-1}{\|K\|_{\infty}^{1 / 2}}$.

Note that, to prove Proposition 3.2 we can suppose $\phi \leq \rho_{e}$ on $\Sigma$.

Proof. We show that $\phi$ satisfies the minimum principle on $\Sigma$. On the contrary, suppose

$$
\gamma:=i n f_{\Sigma} \phi<\delta
$$

there exists a sequence of points in $\left(p_{n}\right)_{n \in \mathbb{N}} \in \Sigma$ such that $\lim _{n} \phi\left(p_{n}\right)=\gamma$.

Take an $\epsilon_{1}$-tube $T\left(\epsilon_{1}, \Sigma\right)$ of $\Sigma$ with $\epsilon_{1}<\rho_{e}$ and consider the restriction of the section defined by $\phi$ that lies in $T\left(\epsilon_{1}, \Sigma\right)$. In other words consider the subdomain $\Sigma_{1} \subset \Sigma$ on which $\phi \leq \epsilon_{1}$.

From Proposition 2.3 it follows that

$$
D_{1}:=\left\{x \in \Sigma_{1} \| \nabla \phi \mid \leq \eta\right\}
$$

if the $\eta$ is sufficiently small. is weakly stable.

We claim that $p_{n}$ lies in $D_{1}$ for $n$ large. Suppose this is not true. Then, $|\nabla \phi|>c$ for some positive $c$.

First there exists an $\mu$-tube $T\left(\partial \Sigma_{1}\right)$ of $\partial \Sigma_{1}$ such that $p_{n} \notin T\left(\partial \Sigma_{1}\right)$. Indeed at these points, the gradient is bounded, so if the distance to the boundary tends to zero the value at these points tends to the value on the boundary, which is strictly bigger than $\gamma$. Hence at each point of $p_{n}$ the ball of radius $\eta / 2$ around $p_{n}$ is inside $\Sigma$.

Second, because the curvature is bounded, there would exist a point $p_{n}^{\prime}$ in $B\left(p_{n}^{\prime}, \eta / 2\right)$ whose $\phi$-value would be strictly less than $\gamma$. This is a contradiction.

Therefore the limit of $\nabla \phi\left(p_{n}\right)$ is zero. Then $\left(p_{n}\right)$ belongs to $D_{1}$ for $n$ large. From Proposition $2.5 D_{1}$ is parabolic, thus $\gamma \geq i n f_{\partial D_{1}} \phi$. But the boundary of $D_{1}$ is composed of points of $\partial \Sigma_{1}$ or points where the gradient has a norm equal to $\eta / 2$. Hence $i n f_{\partial D_{1}} \phi>\gamma$, and $(*)$ is impossible. Finally this is true for any $\epsilon_{1}<\rho_{e}$. Proposition 3.2 follows. 
A consequence of Proposition 3.2 will be:

Corollary 3.3. Let $\Sigma$ and $\Sigma^{\prime}$ be two disjoint properly embedded complete minimal surfaces with nonempty boundary and bounded curvature in a complete flat 3-manifold; then $\operatorname{dist}\left(\Sigma, \Sigma^{\prime}\right)=\min \left(\operatorname{dist}\left(\partial \Sigma, \Sigma^{\prime}\right), \operatorname{dist}\left(\partial \Sigma^{\prime}, \Sigma\right)\right)$.

The argument of the proof of this deduction proceeds as in $[\mathrm{S}]$ where we deduced a weaker version of this corollary (assuming $\Sigma$ to be parabolic) and we will use only Proposition 3.2 here (see [S] for a proof of corollary (3.3)).

\section{Uniform Area Bound and Area Growth.}

3.1. Embedded tubes around $M \in \mathcal{M}$.

A direct consequence of Proposition 3.2 is

Theorem 2. Let $M \in \mathcal{M}$, then there exists a tube of constant radius that is embedded in the Euclidean space. The radius is at least equal to $\rho_{e}$.

Proof. Suppose the $\rho_{e}$-tube around $M$ is not embedded. Then $M$ cuts $T\left(\rho_{e}, M\right)$ away from the zero section. This intersection is a piece of minimal surface, and from estimate (2.8), it is even a graph $\phi$ over a subdomain $M^{\prime}$ of $M$. But then we apply Proposition 3.2 to $\phi$. This gives $\phi \geq \rho_{e}$ because $\left.\phi\right|_{\partial M^{\prime}}=\rho_{e}$. As a result, the $\rho_{e}$-tube of $M$ only contains the zero section of $M$ This establishes Theorem 2 .

\subsection{Uniform Area Bound.}

Let $M$ be a compact piece of minimal surface in $\mathcal{M}$. Let $T(\epsilon, M)$ an $\epsilon$-tube around $M$ with $\epsilon \leq \rho_{e}(*)$. From Theorem $2 T(\epsilon, M)$ is embedded in $\mathbf{E}^{3}$. Thus by Weyl's formula

$$
\operatorname{Vol}(T(\epsilon, M))=2 \epsilon A(M)+\frac{2 \epsilon^{3}}{3} \int_{M} K d s .
$$

In particular

$$
\operatorname{Vol}(T(\epsilon, M)) \geq 2 \epsilon A(M)\left(1-\frac{2 \epsilon^{2}\|K\|^{1 / 2}}{3}\right)
$$

From $(*)$,

$$
\operatorname{Vol}(T(\epsilon, M)) \geq \frac{4}{3} \epsilon A(M)
$$


Thus an upperbound on the volume of the tube of $M$ will furnish un upperbound for the area of any $M \in \mathcal{M}_{1}$.

Now let $S \subset \mathbf{E}^{3}$ be a domain. Then the $\epsilon$-tube around $M \cap S$ is contained in the $\epsilon$-neighbourhood of $S$, hence

$$
\operatorname{Vol}(T(\epsilon, M \cap S)) \leq \operatorname{Vol}\left(S_{\epsilon}\right) .
$$

With (3.3), this gives

Theorem 3. Any $M \in \mathcal{M}$ has an area-growth that is cubic at most. More precisely, for any domain $S \subset E^{3}$,

$$
\text { Area }(M \cap S) \leq c_{1}\|K\|^{1 / 2} \operatorname{Vol}\left(S_{\epsilon}\right)
$$

with $c_{1}=\frac{3}{4(2 \sqrt{3}-5 / 2)(\sqrt{3}-1)}$ where $S_{\epsilon}$ is an $\epsilon$-neighbourhood of $S$, with $\epsilon \geq$ $\frac{1}{\|K\|^{1 / 2}}$. In particular, the area of $M \cap B(p, r)$ is less than $\pi(r+\epsilon)^{3}\|K\|^{1 / 2}$.

Remark. Define $A\left(M_{r}\right)$ the area of $M_{r}=M \cap B(0, r)$ and let $S\left(M_{r}\right)$ the spherical area.

From Theorem 3:

$$
A\left(M_{r}\right) \leq c_{2}(r+\epsilon)^{3}\|K\|^{1 / 2}
$$

with $c_{2}=\frac{\pi}{(2 \sqrt{3}-5 / 2)(\sqrt{3}-1)}$.

Furthermore we shall deduce from $\S 4$ that

$$
\varlimsup_{r \rightarrow \infty} \frac{S\left(M_{r}\right)}{A\left(M_{r}\right)}=0
$$

From (3.3) we deduce

$$
\varlimsup_{r \rightarrow \infty} \frac{A\left(M_{r}\right)}{c_{3}\|K\|^{1 / 2} r} \leq 1
$$

where $c_{3}=\frac{1}{2(\sqrt{3}-1)}$.

\section{Spherical Area Estimate.}

Theorem 4. The Gauss map $N: M \longrightarrow \mathbf{E}^{3}$ has a spherical area growth that is at most linear: for any $r$-ball around $p \in M$, the spherical area of the connected component of $M$ containing $p$ satisfies $N\left(M_{p} \cap B(p, r)\right) \leq$ $C\|K\|_{\infty} r$, where $C \in \mathbb{R}^{+}$is a constant independent of $M$. 
Proof. Let $M \in \mathcal{M}$. Let $p$ be any point on $M$ and let $r$ the Euclidean distance function taken from $p ; M_{r}$ will denote the connected component of $M$ in $M \cap B(p, r)$ containing $p$. $r$, as a function on $M$, satisfies

$$
\Delta r^{2}=\Delta\langle X, X\rangle=2\langle\Delta X, X\rangle+2\langle\nabla X, \nabla X\rangle .
$$

The metric on $\Sigma$ is induced by the immersion, so: $\langle\nabla X, \nabla X\rangle=2$ and $M$ is minimal so $X$ is harmonic: $\Delta X=0$. Hence

$$
\Delta r^{2}=4
$$

and

$$
\Delta r=\frac{2-|\nabla r|}{r} .
$$

From Stokes formula

$$
\int_{M_{r}} \Delta r d s=\int_{M_{r}} \frac{2-|\nabla r|}{r} d s=\int_{\partial M_{r}}|\nabla r| d l .
$$

From first variation of length

$$
\frac{d}{d r} \int_{\partial M_{r}}|\nabla r| d l=\int_{\partial M_{r}} \kappa_{g} d l+\int_{\partial M_{r}} \nabla_{\frac{\nabla r}{|\nabla r|^{2}}}(|\nabla r|) d l .
$$

Let $S(r)=-\int_{M_{r}} K d s$.

From the co-area formula:

$$
\int_{\partial M_{r}} \nabla_{\frac{\nabla r}{|\nabla r|^{2}}}(|\nabla r|) d l=\frac{1}{2} \cdot \frac{d}{d r}\left(\int_{M_{r}} \nabla_{\frac{\nabla r}{|\nabla r|^{2}}}|\nabla r|^{2} d s\right) .
$$

Define $S(r)=\int_{M_{r}}-K d s$. From Gauss-Bonnet theorem:

$$
\int_{\partial M_{r}} \kappa_{g} d l=S(r)-2 \pi \chi(M)=S(r)-2 \pi .
$$

because $M_{r}$ is simply-connected. Hence we use (4.1) and obtain from (4.2)

$$
\frac{d}{d r} \int_{M_{r}} \frac{2-|\nabla r|}{r} d s=S(r)-\frac{1}{2} \frac{d}{d r}\left(\int_{M_{r}} \nabla \frac{\nabla r}{|\nabla r|^{2}}|\nabla r|^{2} d s\right) .
$$

Furthermore

$$
\frac{d}{d r}\left\langle\nabla_{\nabla r} \nabla r, \nabla r\right\rangle=\operatorname{Hess}_{M}(r)(\nabla r, \nabla r) .
$$


We compute Hess $s_{M}$ in terms of the Hessien in $\mathbf{E}^{3}$;

$$
\operatorname{Hess}_{E^{3}}(r)(\nabla r, \nabla r)=\operatorname{Hess}_{M}(r)(\nabla r, \nabla r)+\left\langle A(\nabla r, \nabla r), \nabla^{\perp} r\right\rangle .
$$

But

$$
\operatorname{Hess}_{E^{3}}(r)(\nabla r, \nabla r)=\frac{|\nabla r|^{2}-|\nabla r|^{4}}{r} .
$$

We directly deduce the bound :

$$
\nabla_{\frac{\nabla r}{|\nabla r|^{2}}}|\nabla r|^{2} \leq 2\left|\nabla^{\perp} r\right||A|+\frac{2\left|\nabla^{\perp}\right|^{2}}{r} .
$$

We now plug (4.4) into (4.3), and integrate with respect to $r$; this gives the following bound on the spherical area $\left(|\nabla r|^{2}+\left|\nabla^{1_{r}}\right|^{2}=1\right)$ :

$$
\int_{0}^{r} S(t) d t \leq \int_{M_{r}}\left(\frac{1+|\nabla r|^{2}}{r}+|A|\right) d s+2 \pi r
$$

From Cauchy-Schwarz formula:

$$
\int_{0}^{r} S(t) d t \leq \int_{M_{r}} \stackrel{2}{2} d s+S(r)^{1 / 2} A(r)^{1 / 2}+2 \pi r .
$$

Define $\dot{\psi}(r)=\int_{0}^{r} S(t) d t$. Integration by parts on the first factor of the right hand term gives the following differential inequality:

$$
\psi(r) \leq 2 \frac{A(r)}{r}+\int_{0}^{r} \frac{A(t)}{t^{2}} d t+\psi(r)^{1 / 2} A(r)^{1 / 2}+2 \pi r
$$

From Theorem $3 A(r) \leq c r^{3}$; hence

$$
\psi(r) \leq \frac{5}{2} c r^{2}+c^{\frac{1}{2}}\left(\psi^{\prime}(r) ;\right)^{\frac{1}{2}} r^{\frac{3}{2}}+2 \pi r^{2}
$$

First let us show that $\psi$ has subquadratic growth ; it suffices to consider the differential inequality

$$
\psi(r)^{2} \leq c \psi^{\prime}(r) r^{3}
$$

Hence

$$
\frac{1}{c r^{3}} \leq \frac{\psi^{\prime}(r)}{\psi^{2}(r)}
$$

Integrate from $r$ to $\infty$ using $\lim _{r \rightarrow \infty} \psi(r)=\infty$;

$$
\psi(r) \leq 2 c r^{2}
$$

But $\psi=\int S(t) d t$ with $S$ increasing. Hence,

$$
S(r) \leq 4 c r
$$

This proves Theorem 4. 


\section{5. $\mathcal{M}$ is compact.}

In the last paragraph, we establish the strong compactness of $\mathcal{M}$. This property is a direct consequence of the uniform area bound stated in Section 3.

Any two surfaces of $\mathcal{M}$ will be identical if one is carried onto the other by either a rigid motion or a homothety of $\mathbf{E}^{3}$. Thus we can normalize the curvature function $K$ of any $M \in \mathcal{M}$ such that: $-1 \leq K \leq 0$. In this context, we have

Theorem 5. For any sequence of minimal surfaces in $\mathcal{M}$, with at least one accumulation point in $\mathbf{E}^{3}$, a subsequence converges to a $M \in \mathcal{M}$ with multiplicity one in all compact sets of $\mathbf{E}^{3}$.

Proof. Let $\left(M_{n}\right) \in \mathcal{M}$ a subsequence of embedded minimal minimal surfaces with an accumulation point in $\mathbf{E}^{3}$. From Theorem 2, $M_{n}$ are proper.

Then it follows from Theorem 3. that for any compact domain $K$ of $\mathbf{E}^{3}$, the area of $M_{n} \cap \Omega$ is bounded independently from $n$ : there is a constant $c_{\Omega}$ such that

$$
A\left(M_{n} \cap \Omega\right) \leq c_{\Omega} .
$$

Since $K$ is uniformly bounded, it follows from 5.1 that there exists another constant $c_{\Omega}^{\prime}$ that bounds the spherical area:

$$
S\left(M_{n} \cap \Omega\right) \leq c_{\Omega} .
$$

We are now in a position to apply results of H.I. Choi, R Schoen, and B. White (cf. [CS], [W]) as stated by A. Ros in [R]. There exists a subsequence $\left(M_{n_{k}}\right)$ if $\left(M_{n}\right)$, a properly embedded minimal surface $M \in \mathbf{E}^{3}$ and a discrete singular set $X \subset M$ such that: for any $p \in M \backslash X$ and any positive $\epsilon$, there exists a cylinder $C_{p}(\epsilon)$ around $\mathrm{p}$, of finite height and normal to $M$ such that $C_{p}(\epsilon) \cap M_{n_{k}}$ is a multi-valued graph of finite multiplicity above $M \cap C_{p}(\epsilon)$. Moreover this graph converges in the $C^{k}$-norm to $M \cap C_{p}(\epsilon)$ as $n_{k}$ tends to infinity.

If $p \in X$, then necessarily

$$
\varlimsup_{n} S\left(M_{n} \cap B(p, \epsilon)\right) \geq 4 \pi
$$

for any $\epsilon \in \mathbb{R}^{+}$. 
However the spherical area estimate given in Theorem 4 implies that $X$ is empty. Furthermore, from Theorem 2 we conclude that the multiplicity of convergence is one.

$C^{2}$-convergence implies that the curvature function of $M$ is bounded by one, thus $M \in \mathcal{M}$.

\section{References.}

[AB] L. Ahlfors and A. Beurling, Conformal invariants and functiontheoric null sets, Acta Math., 83 (1950), 101-129.

[CY] S.Y. Cheng and S.T. Yau, Differential Equations on Riemannian Manifolds and their applications, Comm. Pure \& Appl. Math., 28 (1975), 333-354.

[CS] H.I. Choi and R. Schoen, The space of minimal embeddings of a surface into a 3-manifold of positive Ricci curvature, Inv. Math., 81 (1985), 387-394.

[C] Q. Chen, On the total curvature and area growth of minimal surfaces in $R^{n}$, Manu. Math., 92 (1997), 135-142.

[FC] D. Fischer-Colbrie, On complete minimal surfaces with finite Morse index in three manifolds, Inventiones Math., 82 (1985), 121-132.

[FCS] D.Fischer-Colbrie and R. Schoen, The structure of complete minimal surfaces in 3-manifolds of non-negative scalar curvature, Comm. in Pure \& App. Math., 33 (1980), 199-211.

[GT] D. Gilbarg and N. Trudinger, Elliptic partial differential equations of second order, Springer Verlag, 33 (1983).

[H] A. Huber On subharmonic functions and differential geometry in the large, Comment. Math. Helv., 32 (1957), 13-72.

[RR] L. Rodriguez and H. Rosenberg, Some remarks on complete simply connected minimal surfaces meeting the planes $x_{3}=$ cste transversally, preprint, 1996.

[R] A. Ros, Compactness ofd spaces of properly embedded minimal surfaces with finite total curvature, Ind. Univ. Math. J., 44 (1995), 99151. 
[SS] L. Simon and R. Schoen, Seminar on Minimal Submanifolds, Princeton University Press, Princeton, 1983.

[S] M. Soret, Maximum Principle at infinity, Annals of Global Analysis and Geometry, 13 (1995), 101-116.

[WA] G.N. Watson, A treatise on the theory of Bessel Functions, Cambridge University Press, Cambridge, 1980.

[W] B. White Curvature estimates and compactness theorems in 3-manifolds for surfaces that are stationary for parametric elliptic functionals, Inv. Math., 88 (1987), 243-256.

[X] F. Xavier, Why no new complete simply-connected embedded minimal surfaces have been found since 1776, preprint, 1997.

Université F. Rabelais, DÉP. De Mathématiques

37200 TOURS, France

E-mail address: macr@gargan.math.univ-tours.fr

ReCeived April 29, 1998. 